\title{
Influence of concrete strength fractile estimation on the safety level of existing structures assessed using the Adjusted Partial Factor Method
}

\author{
Robby CASPEELE \\ Postdoctoral Researcher \\ Ghent University \\ Ghent, Belgium \\ Robby.Caspeele@UGent.be \\ Robby Caspeele, born 1983 , \\ received his civil engineering \\ degree from the Univ. of Ghent in \\ 2006. In 2010 he there received \\ his $\mathrm{PhD}$ degree. Currently he is \\ employed as a postdoctoral \\ researcher.
}



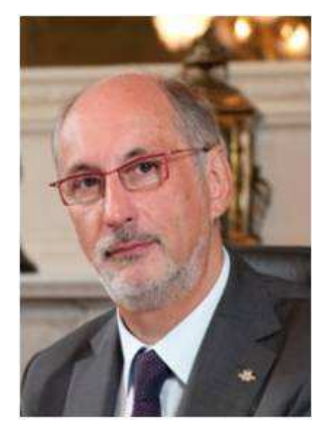

\section{Summary}

In case of existing concrete structures, the estimation of the characteristic strength values from limited data is a difficult, but important task. There are currently different assessment methods available, among which the classical coverage method, a Bayesian procedure with vague prior distributions (as mentioned in EN 1990) and the method described in EN 13791. With respect to the assessment of existing structures, the authors have developed and investigated an Adjusted Partial Factor Method, which is compatible with current Eurocodes, but additionally enables to incorporate alternative target reliability levels and reference periods and also additional information based on e.g. on-site inspection data and data from testing. In this contribution the influence of the different fractile estimation methods on the safety level of concrete structures is investigated, considering the Adjusted Partial Factor Method. The performance of the different estimation methods are evaluated and compared using Monte Carlo simulations and FORM analyses.

Keywords: existing structures, concrete, assessment, partial factors, safety level

\section{Introduction}

In contrast to the design of new structures, the assessment of existing structures often relies on the subjective judgement of the investigating engineer. However, in a previous analysis the authors have showed that an objective verification format for existing structures is feasible and they proposed an Adjusted Partial Factor Method for existing structures [1,2], enabling a rather simple and straightforward, but objective and coherent safety evaluation of existing concrete structures by practitioners. The proposed framework is compatible with the current Eurocodes for the design of new structures, but additionally enables to incorporate alternative values for the target reliability level, alternative values for the reference period and also updated information based on e.g. on-site inspection data and data from testing, as these all considerably influence the partial factors in the structural reliability assessment of existing structures.

In case of concrete structures, the estimation of the characteristic strength values from limited data is a difficult, but important task when assessing the performance of existing structures. There are currently different assessment methods available in literature. First of all, the classical coverage method can be used for this assessment of the characteristic in-situ compressive strength $f_{c k, i s}$ from $n$ test results. Otherwise, based on the standards ISO 12491 [3], ISO 13822 [4] and ISO 2394 [5], the characteristic strength may also be determined using a prediction method which is referred to as a 'Bayesian procedure with vague prior distributions', which is also incorporated in the European Standard EN 1990 [6]. Finally, the assessment can also be based on the European standard EN 13791 [7], which considers criteria which are closely linked to conformity assessment.

These estimation methods inherently result in an additional uncertainty with respect to the parameters of probabilistic models used in risk analyses and as such these phenomena are important to consider when comparing the risk-based performance of partial factor methods for existing structures. Although specifically applied to concrete strength, this contribution provides an original 
framework for combining the performance of estimation methods and structural risk analyses, which can also be used for other structural parameters which have to be assessed when dealing with existing structures.

\section{Available fractile estimation methods}

\subsection{The classical coverage method}

According to the classical coverage method the lower $\alpha$ fractile estimate $\hat{x}_{\alpha}$ is determined in such a way that the probability that the estimated $\hat{x}_{\alpha}$ is lower that the exact fractile $x_{\alpha}$ is equal to a chosen confidence level $\gamma$, which yields Eq. (1). This confidence level $\gamma$ is often assumed to be 0,75 , 0,90 or $0,95[8]$.

$$
P\left[\hat{x}_{\alpha} \leq x_{\alpha}\right]=\gamma
$$

If concrete strength is considered as a normally distributed variable and the coefficient of variation $\delta_{X}$ is unknown, the sample standard deviation $s_{X}$ needs to be calculated and the estimated value $\hat{f}_{c k, i s}$ is calculated as:

$\hat{f}_{c k, i s}=\bar{x}\left(1-\lambda d_{X}\right)$

with $\lambda$ a coefficient depending on $n$ and $\gamma$ and $d_{X}=s_{X} / \bar{x}$ the sample coefficient of variation.

Hence, the coefficients $\lambda$ depend on the sample size $n$ and on the confidence level $\gamma$. The most important advantage of this method is the explicit knowledge of $\gamma$, which is the probability that the estimate $\hat{f}_{c k, i s}$ will be on the safe side of the actual value $f_{c k, i s}$. To take account of statistical uncertainties, a value of $\gamma=0,75$ is recommended for this case in ISO 2394 [5]. However, when an unusual reliability level is required, a higher confidence level of 0,95 would be more appropriate [8].

Table 1 provides an overview of the coefficients $\lambda$ in case this coverage method is used and the coefficient of variation is unknown.

Table 1: $\lambda$ values for the classical coverage method with unknown coefficient of variation [8]

\begin{tabular}{c|cccccccc}
\hline & \multicolumn{10}{c}{$n$} \\
\cline { 2 - 9 } & 3 & 4 & 5 & 6 & 7 & 10 & 20 & $\infty$ \\
\hline$\gamma=0,75$ & 3,15 & 2,68 & 2,46 & 2,34 & 2,19 & 2,10 & 1,93 & 1,64 \\
$\gamma=0,90$ & 5,31 & 3,96 & 3,40 & 3,09 & 2,75 & 2,57 & 2,21 & 1,64 \\
$\gamma=0,95$ & 7,66 & 5,14 & 4,20 & 3,71 & 3,19 & 2,91 & 2,40 & 1,64 \\
\hline
\end{tabular}

\subsection{Bayesian method with vague prior information (EN1990)}

In Annex D of the European Standard EN 1990 [6], which is in agreement with the International Standards ISO 2394 [3], ISO 12491 [4] and ISO 13822 [5], an estimation method is mentioned, which is called a 'Bayesian prediction method with vague prior distributions'. Based on the assumption of a normal distribution, this method estimates the characteristic concrete strength with the same equations as for the classical coverage method. So, the characteristic strength is estimated based on the same Eq. (1) in case the coefficient of variation is unknown.

The parameter $\lambda$, however, has different values compared to those which were mentioned in the previous section. Based on classical Bayesian statistics, the coefficient $\lambda$ in case of an unknown coefficient of variation can be shown to be: 


$$
\lambda=\left|t_{n-1 ; 0,05}\right| \sqrt{1+\frac{1}{n}}
$$

with $\lambda=t_{n-1 ; 0.05}$ the $5 \%$ fractile of the $t$-distribution with $n-1$ degrees of freedom.

A major advantage of this Bayesian method is that no arbitrary assumptions (such as the choice of a confidence level $\gamma$ ) are needed, as was the case in the previously mentioned classical coverage method. Instead, all relevant information obtained by the test results is used in order to update the vague prior information and based on this updated distribution, the characteristic value is determined.

Table 2 provides an overview of the coefficients $\lambda$ in case of this Bayesian method with vague priors. These values are also provided in the European Standard EN 1990 [6].

Table 2: $\lambda$ values in case of a Bayesian method with vague priors and unknown $\delta_{X}[8]$

\begin{tabular}{r|cccccccc}
\hline$n$ & 3 & 4 & 5 & 6 & 7 & 10 & 20 & $\infty$ \\
\hline$\delta_{X}$ unknown & 3,37 & 2,63 & 2,33 & 2,18 & 2,00 & 1,92 & 1,76 & 1,64 \\
\hline
\end{tabular}

Note that in case of an infinite number of test samples, these values again reduce to the theoretical value of 1.64. Further, note that the $\lambda$ values, in case $\delta_{X}$ is unknown, are comparable with the $\lambda$ values in case of the coverage method with $\gamma=0,75$, although the statistical background of both methods is different [9].

\subsection{The EN 13791 method}

The European Standard EN 13791 [7] describes yet another estimation method for the in-situ characteristic compressive strength of concrete. The background of the prescribed method is unclear, but the method seems closely correlated to the conformity criteria of EN 206-1 [10], although these have a completely different purpose [11].

The method described in EN 13791 distinguishes 2 statistical procedures, namely in case 15 or more test results are available (approach A) and in case 3 to 14 test results are available (approach B). In case 15 or more test results are available (approach A), $f_{c k, i s}$ is estimated by:

$$
\hat{f}_{c k, i s}=\min \left\{\bar{x}-k_{2} s ; x_{\min }+4\right\}
$$

with $s$ the sample standard deviation with a minimum value of $2 \mathrm{MPa}, k_{2}$ a coefficient given by national provisions or - if no value is given - taken as 1,48 and $x_{\min }$ the lowest in-situ compressive strength test result.

In case only 3 to 14 test results are available (approach B), $f_{c k, i s}$ is estimated by:

$$
\hat{f}_{c k, i s}=\left\{\bar{x}-k ; x_{\min }+4\right\}
$$

with $k=7$ in case $3 \leq n \leq 6, k=6$ in case $7 \leq n \leq 9$ and $k=5$ in case $10 \leq n \leq 14$.

Note that in case $n>15$ still a parameter value of 1,48 is used and that this value does not converge to the theoretical value of 1,64 as was the case for the 2 previously described methods. This is due to the completely different design philosophy which was used for the derivation of the parameters for the conformity criteria in EN 206-1. An improvement with respect to the performance of the estimation method was analyzed in $[11,12]$ considering the use of approach A also when less than 15 test results are available and to base appropriate $k_{2}$ coefficients for values of $n<15$ on the same conformity control design principles as used for the derivation of the value 1,48. Appropriate values can e.g. be found in [11-13], e.g. resulting in the use of $k_{2}=2,67$ in case of $n=3$ test results. 


\section{The Adjusted Partial Factor Method}

The basic philosophy of the Adjusted Partial Factor Method consists of calculating an adjusted partial factor $\gamma_{X \text {,exist }}$ for existing structures, considering alternative values for the reference period $t_{r e f}$, the target reliability index $\beta$ and the coefficient of variation $\delta_{X}$ of the variable under

consideration. For a given variable $X$ this partial factor is established by simply multiplying the partial factor $\gamma_{X}$ as provided in the Eurocodes for new structures by an adjustment factor $\omega_{\gamma}$, i.e.:

$$
\gamma_{X, \text { exist }}=\omega_{\gamma}\left(t_{r e f}, \beta, \delta_{X}\right) \cdot \gamma_{X}
$$

Due to the fact that the method recognizes the target reliability level as an input parameter, the proposed framework remains valid under different human life and economic cost-optimization considerations (i.e. under different national or international economic considerations). Suitable methods for decision making related to the target reliability index can e.g. be found in [14-17].

Considering the Simplified Level II method developed by König \& Hosser [18] for the derivation of partial factors for a semi-probabilistic Level I design format, which is also included in EN 1990, equations can be suggested for the adjustment factor $\omega_{\gamma}$. Consequently a set of easy-to-use graphs can be generated, which is an importance advantage for the practical applicability of the proposed design format by practitioners. For these graphs, reference is made to previous publications by the authors in $[1-2,19]$. An overview of the proposed adjustment factors is given in the following table.

Table 3: Adjustment factors for material properties, permanent and variable actions

\begin{tabular}{ll}
\hline Variable & Adjustment factor \\
\hline $\begin{array}{l}\text { Material } \\
\text { property } X\end{array}$ & $\omega_{\gamma}=\frac{\gamma_{R d, X}\left(\beta^{\prime \prime}\right)}{\gamma_{R d, X}\left(\beta^{\prime}\right)} \exp \left(\alpha_{R} \beta^{\prime} \delta_{X}^{\prime}\left(\frac{\beta^{\prime \prime}}{\beta^{\prime}} \frac{\delta_{X}^{\prime \prime}}{\delta_{X}^{\prime}}-1\right)-1,645 \delta_{X}^{\prime}\left(\frac{\delta_{X}^{\prime \prime}}{\delta_{X}^{\prime}}-1\right)\right)$ \\
$\begin{array}{l}\text { Permanent } \\
\text { actions }\end{array}$ & $\omega_{\gamma}=\frac{\gamma_{E d, G}\left(\beta^{\prime \prime}\right)}{\gamma_{E d, G}\left(\beta^{\prime}\right)} \cdot \frac{1-\alpha_{E} \beta^{\prime \prime} \delta_{G}^{\prime \prime}}{1-\alpha_{E} \beta^{\prime} \delta_{G}^{\prime}}$ \\
$\begin{array}{l}\text { Imposed } \\
\text { loads }\end{array}$ & $\omega_{\gamma}=\frac{\gamma_{E d, Q}\left(\beta^{\prime \prime}\right)}{\gamma_{E d, Q}\left(\beta^{\prime}\right)} \frac{1+\delta_{5}^{\prime \prime}\left[0,78 \ln \left(t_{r e f}^{\prime \prime} / 5\right)-0,45-0,78 \ln \left(-\ln \left(\Phi\left(-\alpha_{E} \beta^{\prime \prime}\right)\right)\right)\right]}{1+\delta_{5}^{\prime}\left[0,78 \ln \left(t_{r e f}^{\prime} / 5\right)-0,45-0,78 \ln \left(-\ln \left(\Phi\left(-\alpha_{E} \beta^{\prime}\right)\right)\right)\right]}$ \\
$\begin{array}{l}\text { Climatic } \\
\text { actions }\end{array}$ & $\omega_{\gamma}=\frac{\gamma_{E d, Q}\left(\beta_{\text {clim }}^{\prime \prime}\right)}{\gamma_{E d, Q}\left(\beta_{\text {clim }}^{\prime}\right)} \cdot \frac{1+\delta_{1}^{\prime \prime}\left[0,78 \ln t_{r e f}^{\prime \prime}-0,45-0,78 \ln \left(-\ln \left(\Phi\left(-\alpha_{E} \beta_{\text {clim }}^{\prime \prime}\right)\right)\right)\right]}{1+\delta_{1}^{\prime}\left[0,78 \ln t_{r e f}^{\prime}-0,45-0,78 \ln \left(-\ln \left(\Phi\left(-\alpha_{E} \beta_{\text {clim }}^{\prime}\right)\right)\right)\right]}$ \\
\hline
\end{tabular}

In Table 3 the characteristics with respect to new structures are designated with ' and those for existing structures with ", $\beta$ is the reliability index (allowing for an alternative target value $\beta_{\text {clim }}$ for climatic actions), $t_{\text {ref }}$ the reference period (most often considered equal to the remaining working life) and $\delta_{X}$ is the coefficient of variation associated to the variable under consideration. Further, the sensitivity factors $\alpha_{R}$ and $\alpha_{E}$ are considered invariant under the adjustment procedure (which is true in case of the Simplified Level II approach) and are commonly chosen 0,8 and $-0,7$, respectively.

The partial factors related to the model uncertainties $\gamma_{R d}, \gamma_{E d, G}$ and $\gamma_{E d, Q}$ mentioned in Table 3 can in general be calculated according to (7) and (8), respectively.

$$
\begin{aligned}
& \gamma_{R d}=1 /\left(1-\alpha_{R} 0,4 \beta_{t} \delta_{\theta}\right) \\
& \gamma_{E d}=1-\alpha_{E} 0,4 \beta_{t} \delta_{\theta}
\end{aligned}
$$

For material properties, it is more common to consider 2 types of model uncertainties, namely probabilistic model uncertainties and geometrical uncertainties, i.e. $\gamma_{R d}=\gamma_{R d 1} \cdot \gamma_{R d 2}$ [20]. The associated coefficients of variation $\delta_{\theta}$ are provided in Table 4 , together with $\delta_{X}^{\prime}$. 
Table 4: Coefficients of variations (COV)

\begin{tabular}{lll}
\hline $\begin{array}{l}\text { Resistance/load } \\
\text { variable }\end{array}$ & $\begin{array}{l}\mathrm{COV} \delta_{\theta} \text { of model uncertainty of } \\
\text { resistance/load effect }\end{array}$ & $\begin{array}{l}\mathrm{COV} \delta_{X}^{\prime} \text { of material property/load } \\
\text { considered for new structures }\end{array}$ \\
\hline Concrete strength & $\delta_{\theta, R d 1, c}=0,075, \delta_{\theta, R d 2, c}=0,075$ & $\delta_{c}^{\prime}=0,15$ \\
Steel yield strength & $\delta_{\theta, R d 1, s}=0,02, \delta_{\theta, R d 2, s}=0,04$ & $\delta_{S}^{\prime}=0,05$ \\
Permanent load & $\delta_{\theta, G}=0,065$ & $\delta_{G}^{\prime}=0,10$ \\
Imposed load & $\delta_{\theta, Q}=0,11$ & $\delta_{Q, 5}^{\prime}=0,45$ \\
Climatic load & $\delta_{\theta, Q}=0,11$ & $\delta_{Q, 50}^{\prime}=0,25$ \\
\hline
\end{tabular}

\section{Influence of fractile estimation on the safety level of existing structures}

A thorough investigation of the numerical performance of the different estimation methods was previously performed by the authors, hence referring to [12]. In the current contribution the influence of the fractile estimation of concrete strength on the structural reliability of concrete columns is assessed using FORM analyses. Consider the following limit state function for a short concrete column subjected to compression:

$$
g(\underline{X})=K_{R}\left(\alpha_{c c} A f_{c}+\rho A f_{y}\right)-K_{E}\left(G+Q_{t r e f}\right)
$$

with $K_{R}$ the resistance model uncertainty, $\alpha_{c c}$ the long-term effect on concrete strength, $A$ the cross-sectional area, $f_{c}$ the concrete strength, $\rho$ the reinforcement ratio, $f_{y}$ the yield strength of the reinforcement, $K_{E}$ the load effect uncertainty, $G$ the permanent load effect and $Q_{\text {tref }}$ the imposed load effect related to a reference period $t_{r e f}$. Probabilistic model characteristics are chosen according to the suggestions of the Joint Committee on Structural Safety [21], considering a reinforcement ratio of $1 \%$, a cross-sectional area of $0,6 \times 0,3 \mathrm{~m}^{2}$, a concrete strength class $\mathrm{C} 30 / 37$ and reinforcement steel S500. For more detailed information about the specific probabilistic model reference is made to [2].

In case the fundamental load combination $(6.10 \mathrm{a}, \mathrm{b})$ according to EN 1990 is used to assess the column strength under compression, the verification criterion can be written as:

$\alpha_{c c} b h \frac{f_{c k}}{\gamma_{c}}+\rho b h \frac{f_{y k}}{\gamma_{s}}=\max \left\{\gamma_{G} G_{k}+\psi_{0, Q} \gamma_{Q} Q_{k} ; \xi \gamma_{G} G_{k}+\gamma_{Q} Q_{t r e f}\right\}$

with $\gamma_{G}$ the partial factor for the permanent load, i.e. 1,$35 ; G_{k}$ the characteristic value of the permanent load effect; $\psi_{0, Q}$ a factor for the combination value of the imposed load effect, i.e. 0,7 ; $\gamma_{Q}$ the partial factor for the imposed load, i.e. 1,$5 ; Q_{\text {tref }}$ the characteristic value of the imposed load effect associated to a reference period $t_{r e f}$, and $\xi$ a reduction factor for the permanent load, i.e. 0,85. Rearranging Eq. (10) and introducing the load ratio $\chi=Q_{k} /\left(G_{k}+Q_{k}\right)$, leads to the following permanent load effect value associated to a specific geometry, reinforcement ratio and load ratio:

$$
G_{k}=\frac{\alpha_{c c} b h f_{c k} / \gamma_{c}+\rho b h f_{y k} / \gamma_{s}}{\max \left\{\gamma_{G}+\psi_{0, Q} \gamma_{Q} \frac{\chi}{1-\chi} ; \xi \gamma_{G}+\gamma_{Q} \frac{\chi}{1-\chi}\right\}}
$$

Further, in case of existing structures, the nominal values of the parameters in (10) have to be assessed. In case the concrete strength is estimated for laboratory testing on drilled cores, in (11) $f_{c k}$ has to be replaced by $\hat{f}_{c k}$, defining the estimation error $\zeta$ by the relation $\hat{f}_{c k}=\zeta f_{c k}$. 
In order to establish a probabilistic model for the estimation error $\zeta$ in case of the different estimation methods (in order to include this additional uncertainty in the reliability analysis) Monte Carlo simulations are executed. 100000 samples of the estimated value $\hat{f}_{c k \text {,is }}$ are generated, considering a standard deviation for the concrete strength of $\sigma=5 \mathrm{MPa}$. The results in terms of the mean and standard deviation of the estimation error $\zeta$ in function of the number of test samples $n$ used for the estimation are given in Figs. 1 and 2.

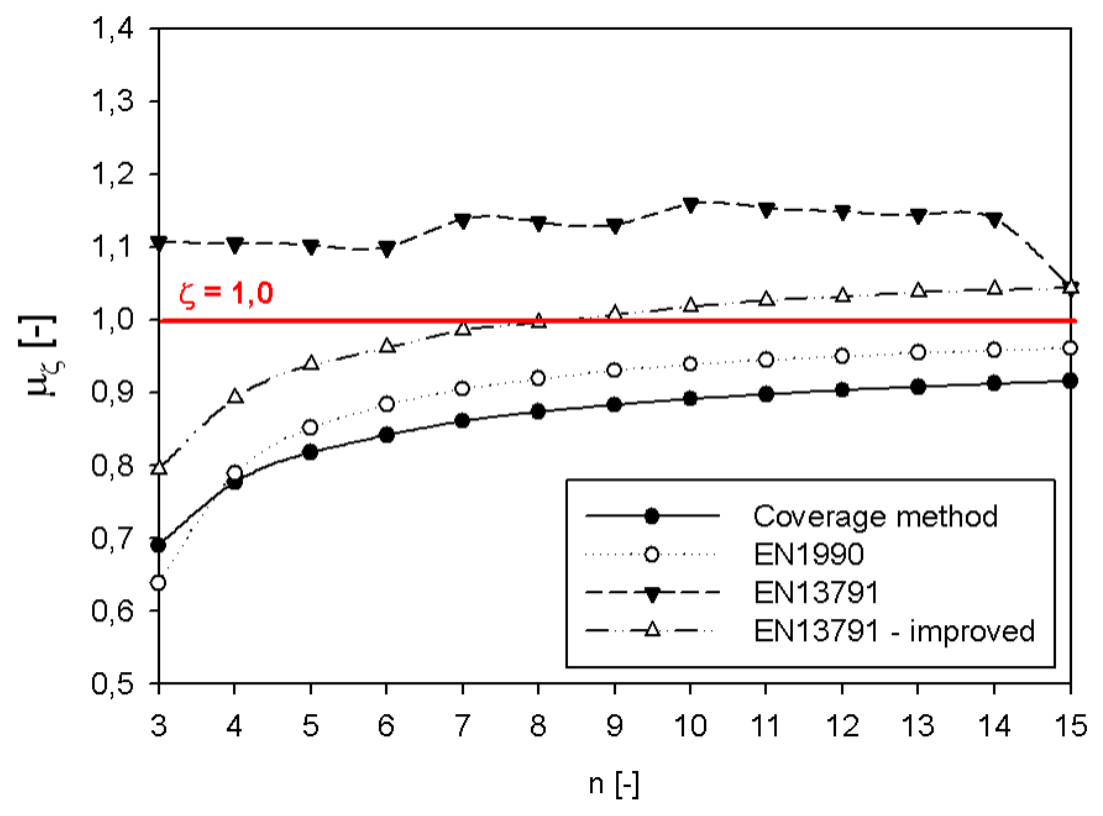

Fig. 1: Influence of the number of test samples on the mean of the estimation error $\zeta$

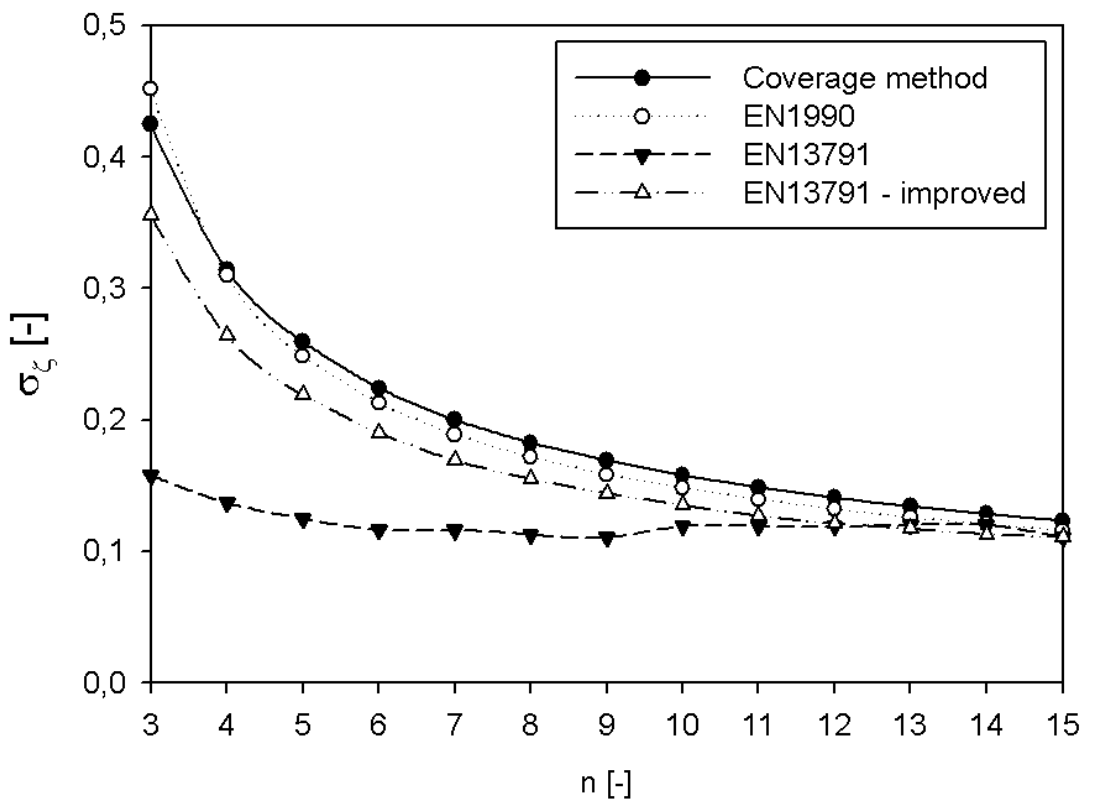

Fig. 2: Influence of the number of test samples on the standard deviation of the estimation error $\zeta$
The classical coverage method and the estimation method according to EN 1990 result in a safe estimation of the characteristic strength, which however is becoming rather conservative in case only a few samples are used for the estimation. On the other hand, the method according to EN 13791 yields an overestimation of the characteristic concrete strength. In case of the latter method, however, the variability of the estimation error is smaller (due to the independence from the sample standard deviation in case less than 15 test results are used).

Further, also the partial factors have to be adjusted in case of existing structures. In this contribution, the suggested Adjusted Partial Factor Method described in section 3 is used for this purpose. The adjustment factor for concrete strength is however dependent on the estimated coefficient of variation, i.e. according to the ratio $\hat{\delta} / \delta^{\prime}$. Similar Monte Carlo simulations are executed in order to establish the mean and standard deviation for this estimated ratio in case of the different estimation methods. Consequently, the mean and standard deviation of the adjusted partial factor for concrete strength can be approximated according to (12) and (13) respectively. 


$$
\begin{aligned}
& \gamma_{c, \text { exist }}=\omega_{\gamma} \gamma_{c}=\gamma_{c} \exp \left(\alpha_{R} \beta^{\prime} \delta_{c}^{\prime}\left(\frac{\beta^{\prime \prime}}{\beta^{\prime}} \frac{\delta_{c}}{\delta_{c}^{\prime}}-1\right)-1,645 \delta_{c}^{\prime}\left(\frac{\delta_{c}}{\delta_{c}^{\prime}}-1\right)\right) \\
& \operatorname{Var}\left[\gamma_{c, \text { exist }}\right]=\left[\gamma_{c}\left(\alpha_{R} \beta^{\prime \prime} \delta_{c}^{\prime}-1,645 \delta_{c}^{\prime}\right) \exp \left(\alpha_{R} \beta^{\prime} \delta_{c}^{\prime}\left(\frac{\beta^{\prime \prime}}{\beta^{\prime}} \frac{\hat{\delta}_{c}}{\delta_{c}^{\prime}}-1\right)-1,645 \delta_{c}^{\prime}\left(\frac{\hat{\delta}_{c}}{\delta_{c}^{\prime}}-1\right)\right)\right]^{2} \sigma_{\delta_{c} / \delta_{c}^{\prime}}^{2}
\end{aligned}
$$

Considering lognormal distributions for $\zeta$ and $\gamma_{c \text {,exist }}$ (with derived characteristics as previously described), a FORM analysis is performed in order to investigate the influence due to the concrete strength estimation from test samples on the structural safety. The results are visualized in Fig. 3 in case $\beta "=3,1$.

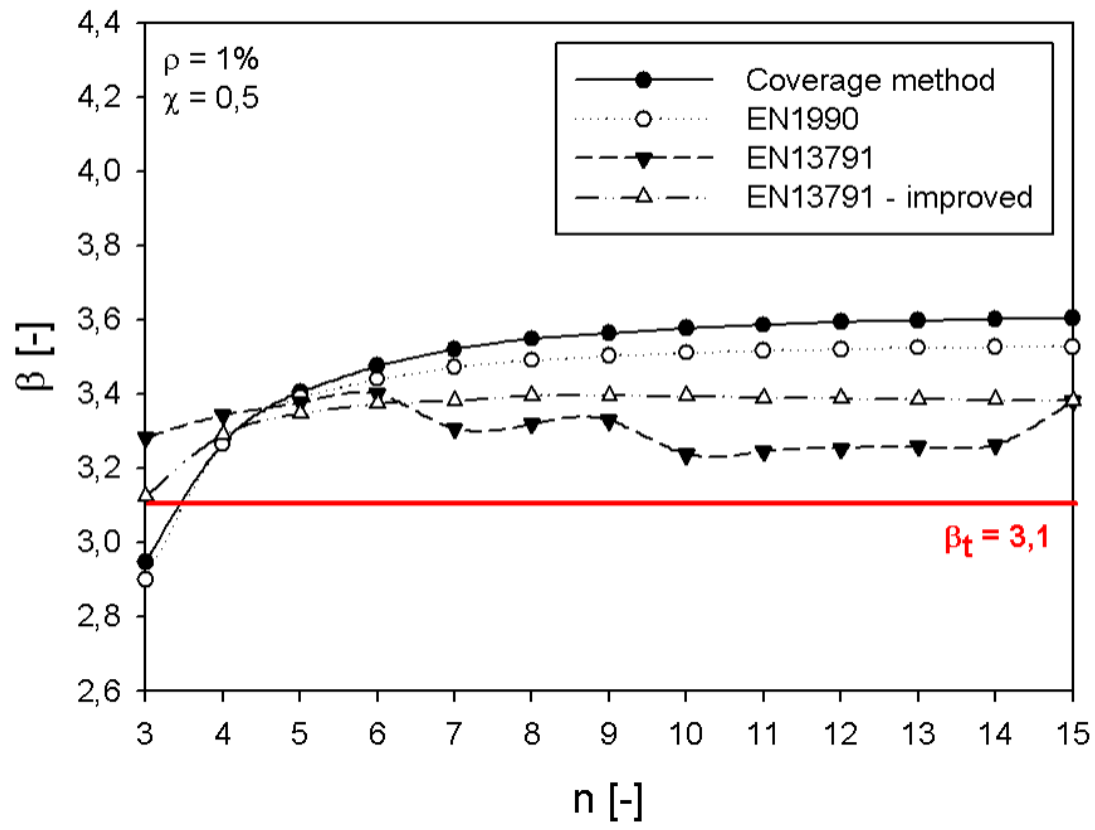

Fig. 3: Influence of the number of test samples on the reliability index of a short concrete column subjected to compression

$$
\text { (in case } \delta_{c}^{\prime \prime} / \delta_{c}^{\prime}=1, \rho=1 \%, \chi=0,5 \text { ) }
$$

The classical coverage method and the EN 1990 method yield a higher safety level. However, in case only a few concrete samples are used for the assessment (i.e. less than 5), the EN 13791 yields a higher safety level, mainly due to the reduced variability with respect to the estimation error. When the variability due to the estimation method is not considered, in this case $\beta$ would be 3,6 . Comparisons in case of $\delta_{c}^{\prime \prime} / \delta_{c}^{\prime}=0,75$ and $\delta_{c}^{\prime \prime} / \delta_{c}^{\prime}=1,25$ result in similar conclusions.

Further simulations proved that the relative performance

of the different estimation methods is not influenced significantly by the assumed value of the standard deviation for the concrete strength. The same observation can be made when an estimation method is used based on a lognormal distribution.

\section{Conclusions}

Currently, there are 3 methods which are commonly used for the estimation of the in-situ characteristic concrete compressive strength. The probabilistic performance of these 3 methods were analyzed and compared using numerical Monte Carlo simulations. Further, these estimations were taken into account in the structural reliability analysis for existing structures, assessed using the Adjusted Partial Factor Method. The performance of the classical coverage method and the EN 1990 method are comparable and yield a higher safety level when more than 5 test samples are considered. However, in case only very few concrete samples are used for the assessment (i.e. less than 5), the EN 13791 yields a higher safety level, mainly due to the reduced variability with respect to the estimation error. Further, the analysis also showed that for the investigated situation, taking more than 8 test samples into account does not lead to an increase in safety level. 


\section{References}

[1] CASPEELE R., and TAERWE L., "Proposal of a semi-probabilistic framework for the evaluation of existing concrete structures", Proc. ESREL2011 Annual Conference, 18-22 Sept. 2011, Troyes, France, 2011, pp. 1941-1948.

[2] CASPEELE R., and TAERWE L., "Evaluation of Bayesian updated partial factors for material properties in existing concrete structures", Proc. IALCCE 2012, 3-6 Oct. 2012, Vienna, Austria, 2012, pp. 797-804.

[3] ISO 12491, Statistical methods for durability control of building materials and components, ISO Standard, 1997.

[4] ISO 13822, Basis for design of structures - Assessment of existing structures, ISO Standard, 2003.

[5] ISO 2394, General principles on reliability for structures, ISO Standard, 1998.

[6] EN 1990, Eurocode 0: Basis of structural design, European Standard, CEN, 2002.

[7] EN 13791, Assessment of in-situ compressive strength in structures and precast concrete components, European Standard, CEN, 2007.

[8] GULVANESSIAN H., CALGARO J.-A., and HOLICKY M., Designers' Guide to EN 1990 Eurocode: Basis of structural design, Thomas Telford, London, 2002.

[9] STRUCK W., "The calculation of one-sided lower tolerance limits (fractiles) in statistical evaluation of measurements (in German)", Materalprüfung, Vol. 9, No. 6, 1967, pp. 218-222.

[10] EN 206-1, Concrete - Part 1: Specification, performance, production and conformity, European Standard, CEN, 2001.

[11] CASPEELE R., Probabilistic Evaluation of Conformity Control and the Use of Bayesian Updating Techniques in the Framework of Safety Analyses of Concrete Structures, $\mathrm{PhD}$ thesis, Ghent University, Ghent, Belgium, 2010.

[12] CASPEELE R., and TAERWE L., "Quantitative comparison of estimation methods for determining the in-situ characteristic concrete compressive strength", Structural Engineering International, Vol. 22, No. 2, 2012, pp. 215-222.

[13] TAERWE L. Aspects of the stochastic nature of concrete strength including compliance control (in Dutch), PhD thesis, Ghent University, Ghent, Belgium, 1985.

[14] HOLICKY M., "Target reliability for temporary structures". Proc. 6th IFED forum on Risk for temporary structures and activities, 26-29 Jan. 2012, Lake Louise, Canada, 2012.

[15] STEENBERGEN R., and VROUWENVELDER T., "Safety philosophy for existing structures and partial factors for traffic loads on bridges", Heron, Vol. 55, No. 2, 2010, pp. 123-139.

[16] SYKORA M., HOLICKY M., and MARKOVA J., "Target reliability levels for assessment of existing structures", Proc. ICASP11, 1-4 Aug. 2011, Zurich, Switzerland, 2011, pp. 10481056.

[17] VROUWENVELDER T., and SCHOLTEN N., "Assessment criteria for existing structures", Structural Engineering International, Vol. 20, No. 1, 2010, pp. 62-65.

[18] KÖNIG G., and HOSSER D., The simplified level II method and its application on the derivation of safety elements for level I, CEB Bulletin no. 147, Lausanne, Switzerland, 1982.

[19] CASPEELE R., STEENBERGEN R., and TAERWE L., "Adjustment of partial factors in case of temporary structures", Proc. 6th IFED forum on Risk for temporary structures and activities, 26-29 Jan. 2012, Lake Louise, Canada, 2012.

[20] FIB, Model Code 2010 - Final Draft, Volume 1, fib Bulletin no. 65, Lausanne, Switzerland, 2010 .

[21] JCSS, Probabilistic Model Code, internet publication, http://www.jcss.ethz.ch, 2001. 